\title{
Multiparametric and semiquantitative scoring systems for the evaluation of mouse model histopathology - a systematic review
}

\author{
Robert Klopfleisch
}

\begin{abstract}
Background: Histopathology has initially been and is still used to diagnose infectious, degenerative or neoplastic diseases in humans or animals. In addition to qualitative diagnoses semiquantitative scoring of a lesion's magnitude on an ordinal scale is a commonly demanded task for histopathologists. Multiparametric, semiquantitative scoring systems for mouse models histopathology are a common approach to handle these questions and to include histopathologic information in biomedical research.

Results: Inclusion criteria for scoring systems were a first description of a multiparametric, semiquantiative scoring systems which comprehensibly describe an approach to evaluate morphologic lesion. A comprehensive literature search using these criteria identified 153 originally designed semiquantitative scoring systems for the analysis of morphologic changes in mouse models covering almost all organs systems and a wide variety of disease models. Of these, colitis, experimental autoimmune encephalitis, lupus nephritis and collagen induced osteoarthritis colitis were the disease models with the largest number of different scoring systems. Closer analysis of the identified scoring systems revealed a lack of a rationale for the selection of the scoring parameters or a correlation between scoring parameter value and the magnitude of the clinical symptoms in most studies.
\end{abstract}

Conclusion: Although a decision for a particular scoring system is clearly dependent on the respective scientific question this review gives an overview on currently available systems and may therefore allow for a better choice for the respective project.

Keywords: Score, Semiquantitative, Histopathology, Colitis, Nephritis, Hepatitis, Encephalitis

\section{Background}

Histopathology has initially been and is still used today to diagnose infectious, degenerative or neoplastic diseases in humans or animals. These qualitative diagnoses are based on a sum of observable changes in the morphology of the analyzed tissue. The cognition of these changes is based on the pattern recognition of the observer and the comparison of these patterns with the known physiologic variation in tissue morphology in the respective species. Decades of experience in veterinary pathology show that this approach allows for reproducible qualitative diagnoses by the observer but can

Correspondence: robert.klopfleisch@fu-berlin.de

Department of Veterinary Pathology, College of Veterinary Medicine, Freie Universität Berlin, Berlin, Germany also be used for semiquantitative scoring of the lesions magnitude, i.e. on an ordinal scale for instance with a low, medium or high grade trichotomy which correlates with the clinical relevance of the lesions.

Absolute quantification of the lesions extent and severity is however difficult since two main problems hamper absolute quantification, i.e. on a rational scale with absolute values of 1, 2, 3 etc., using standard, non-automated histopathology. First, the detection method is not reliable enough. Despite intensive training and attempts to standardize nomenclature and the definition of lesions there are still unresolved issues in terms of interobserver variation which may be acceptable for qualitative and semiquantitative evaluation but not for absolute quantitation [1]. Second, in most circumstances it is impossible to objectively justify the interval between two values, thus a 
read out of histopathologic scoring on a rational scale is impossible.

Image analysis by automated calculation of the tissue area affected or cells present per area have been introduced to overcome this problem. These approaches aim at a reliable and reproducible histopathology read out in a rational scale to allow proper statistical processing and at an exclusion of an observer bias [2]. Image analysis approaches usually use one or only few two dimensional planar sections of the tissue of interest to measure threedimensional objects. This two-dimensional approach thus may also lead to biased results. Stereology, which is based on systematic random sampling and estimates third dimensional information, has been developed to avoid this

Table 1 Semiquantitative scoring systems for murine intestinal disease models

\begin{tabular}{|c|c|c|}
\hline Disease model & Scoring system: parameters (scale width) & Citations \\
\hline \multicolumn{3}{|l|}{ Colitis } \\
\hline DSS-induced [4] & $\begin{array}{c}\text { Proximal }+ \text { middle }+ \text { distal colon: crypt loss }(0-4) \text {, inflammation }(0-3) \text { both quantified by the } \\
\text { affected area }(0-4)\end{array}$ & 718 \\
\hline DSS-induced [5] & Extent, inflammation, necrosis, regeneration (each 3-0) & 321 \\
\hline DSS-induced [6] & Crypt damage, area involved, regeneration (each 0-4), extent (0-3), inflammation (0-3), & 255 \\
\hline DSS-induced [7] & $\begin{array}{l}\text { Crypt loss (0-4), crypt distortion (0-4), epithelial hyperplasia (0-4), inflammation (0-3) each } \\
\text { multiplied by percentage of involved area (0-4) }\end{array}$ & 185 \\
\hline DSS-induced [8] & Hyperplasia $(0-3)$, severity $(0-3)$, ulceration $(0,1)$, area involved $(0-4)$ & 147 \\
\hline DSS-induced [9] & $\begin{array}{c}\text { Severity of inflammation, thickness of inflammation, epithelial damage character, extent of } \\
\text { epithelial damage (each } 0-3)\end{array}$ & 130 \\
\hline DSS-induced [10] & Epithelium (goblet cell/crypt loss), infiltration (each 0-4) & 103 \\
\hline DSS-induced [11] & Infiltration, tissue damage (each 0-3) & 89 \\
\hline TNBS-induced [12] & $\begin{array}{l}\text { Inflammation, loss of goblet cells, vascular density, transmural infiltration, thickening of the } \\
\text { colon wall (together } 0-4 \text { ) }\end{array}$ & 788 \\
\hline TNBS-induced [13] & Crypt distortion, goblet cell loss, acute inflammation, chronic inflammation (each 0-2) & 126 \\
\hline TNBS-induced [14] & $\begin{array}{l}\text { Loss of mucosal architecture, cellular infiltration, muscle thickening (each } 0-3) \text {, crypt abscesses }(0,1) \text {, } \\
\text { goblet cell depletion }(0,1)\end{array}$ & 97 \\
\hline Acetic acid-induced [15] & Inflammation, bleeding, ulcer size, deepness, perforation (together 0-6) & 132 \\
\hline TNBS-induced [16] & $\begin{array}{c}\text { Percentage of area, crypt loss (both } 0-4 \text { ) number of follicles, edema, erosion/ulceration, } \\
\text { infiltration (each } 0-3 \text { ) }\end{array}$ & 57 \\
\hline HLA-B27 transgenic mice [17] & $\begin{array}{l}\text { Inflammation, goblet cell loss, mucosal thickening, submucosal infiltration, architecture loss } \\
\qquad(\text { each } 0-4) \text {, ulcer, crypt abscess (each } 0,1)\end{array}$ & 423 \\
\hline IL10-deficient mice [18] & $\begin{array}{l}\text { Mucosal ulceration, epithelial hyperplasia (both 0-3), Lamina propria mononuclear infiltrate, } \\
\text { Lamina propria neutrophil infiltrate (each 0-2) }\end{array}$ & 423 \\
\hline IL10-deficient mice [19] & $\begin{array}{l}\text { Inflammation/epithelial erosion/ulcers/hyperplasia/crypt abscesses/goblet cell depletion } \\
\text { (together 0-4) multiplied by no. of affected colon segments (1-5) }\end{array}$ & 555 \\
\hline MHC missmatch [20] & $\begin{array}{l}\text { Active inflammation (0.5-3), chronic inflammation (0.5-3), villous architecture (1-3) multiplied by the } \\
\text { affected area (0.5-4) }\end{array}$ & 83 \\
\hline Amebic colitis [21] & Number of amoeba (0-5), ulceration (0-100\%), inflammation (0-5) & 58 \\
\hline \multicolumn{3}{|l|}{ Small intestinal diseases } \\
\hline Clostridial toxicosis [22] & Epithelial damage, hyperemia/edema, neutrophils (each 0-3) & 202 \\
\hline Intestinal ischemia [23] & $\begin{array}{c}\text { Normal mucosa (0), villous edema (1), subepithelial edema (2), epithelium loss at villi sides (3), } \\
\text { denuded villi (4), loss of villous tissue (5), crypt infarction (6), transmucosal infarct (7), } \\
\text { transmural infarct (8) }\end{array}$ & 270 \\
\hline Intestinal ischemia [24] & Mucosal damage, inflammation, hyperemia/hemorrhage (each 0-5) & 72 \\
\hline Jejunitis [25] & $\begin{array}{l}\text { Villous length, villous tips, epithelium, inflammation, crypt loss (each } 0-2 \text { ), crypt abscesses, } \\
\text { hemorrhage (each } 0,1)\end{array}$ & 15 \\
\hline Bacterial ileitis [26] & Hemorrhage, villous atrophy/necrosis, edema, congestion, neutrophils, epithelial necrosis (each 0-3) & 1 \\
\hline \multicolumn{3}{|l|}{ Gastric diseases } \\
\hline Helicobacter-induced [27] & Five areas multiplied by inflammation ( $0-3$ ) & 49 \\
\hline
\end{tabular}

DSS, Dextran sodium sulfate; TNBS, 2,4,6-trinitrobenzene sulfonic acid, MHC, Major histocompatibility complex; HLA-B27, Human Leukocyte Antigen-B27, IL10, Interleukin 10. 
bias [3]. It can therefore be seen as the most sophisticated method for the quantification of histologic information. It is however by comparison a laborious and complex method which is established in only few laboratories.

Semiquantitative scoring systems are therefore still the most widely used methods to include histopathologic information in biomedical research. These scoring systems usually include multiple parameters which are separately quantified on an ordinal scale and finally combined in a total score. Average scores of the different experimental groups can then be compared by non-parametric statistical tests. The selection of the parameters should be based on the scientific hypothesis or question together with the current knowledge on the morphologic outcome of the investigated disease model. It may therefore be useful to design individual scoring system for each study which in the best possible way answers the particular scientific question. Standard scoring systems for specific disease models on the other hand allow for the comparison of the results of different studies.

Several standard scoring systems for different mouse models have been introduced or emerged in the past 20 years. Histopathologists are therefore repeatedly requested by cooperating scientists to evaluate the outcome of animal studies using standard scoring systems or to elaborate project specific scoring systems. The present review is intended to give a comprehensive overview on the currently most commonly used multiparametric, semiquantitative scoring systems for mouse model histopathology.

\section{Results}

Scoring systems for murine intestinal disease models

Eighteen original scoring systems for colitis models could be identified. Most of these scoring systems were designed for dextran sodium sulfate (DSS)-induced colitis models but 2,4,6-trinitrobenzene sulfonic acid (TNBS)-induced colitis and several models of immunopathologic colitis were also used to establish scoring systems (Table 1, Figure 1). Eight scoring systems for DSS-induced colitis fulfilled all required parameters and were included in this review. Generally, all of the paper with original DSS colitis scoring systems had a high citation rate but the scoring system described by Cooper is one of the earliest system with the highest citation number up to date and can therefore be seen as a prototype for DSS-colitis scoring [4]. It separates the colon into three segments which are then scored by the parameters of crypt loss, inflammation and affected area. Although later studies refined and increased the number of histopathologic parameters the value of this initial study is the separation of the colon in three segments and the sophisticated approach to the establishment of the scoring system. Remarkably, parameters in this study were chosen and tested according to their correlation with the clinical symptoms of the mice. This is contrast to the vast majority of scoring systems presented in this review, which only rarely stated the rational for choosing the included parameters and did not perform a correlation with the clinical symptoms.

When comparing all original colitis models it becomes obvious that a wide variety of appellation for the most common parameters inflammation, crypt and surface

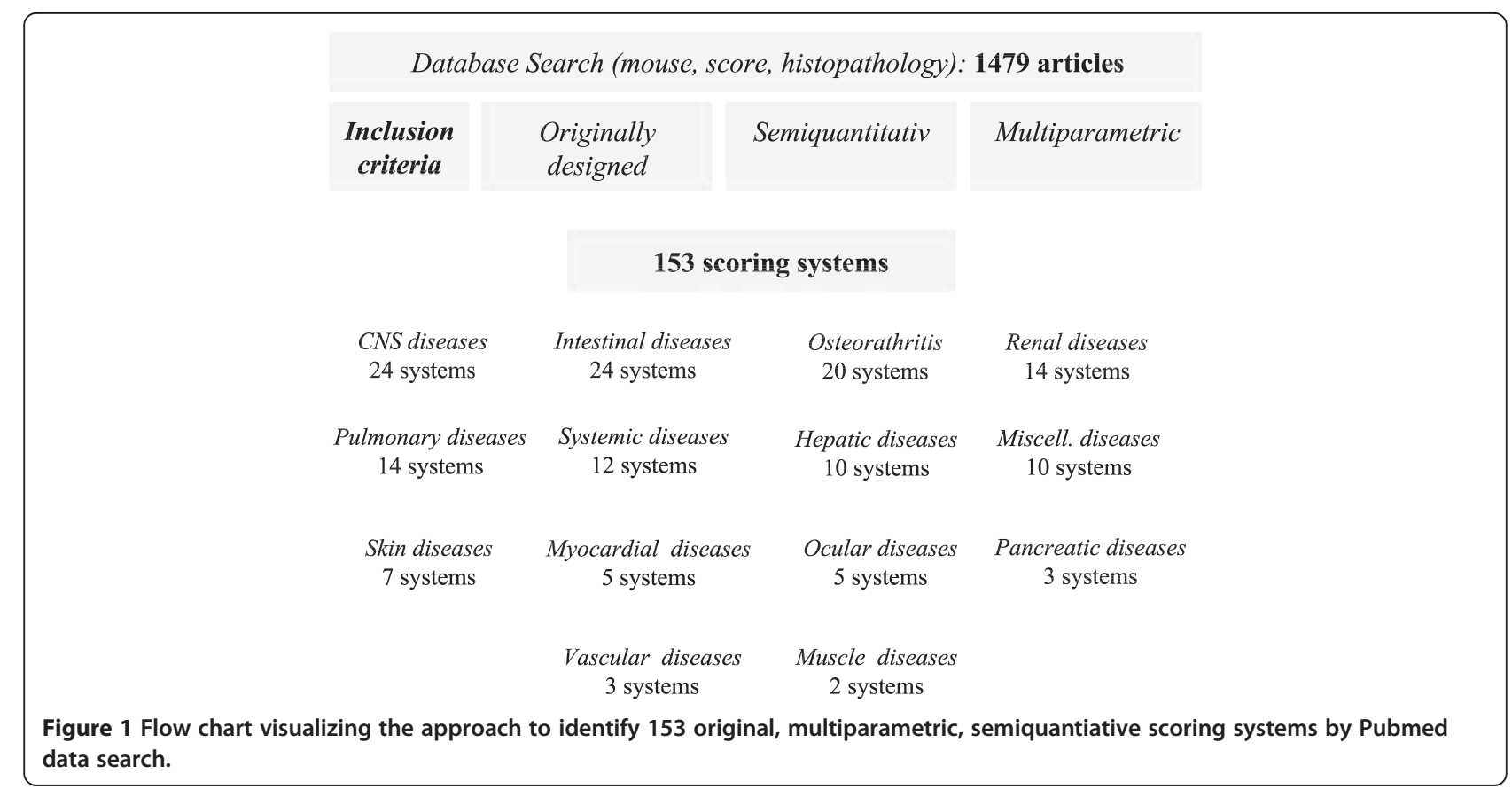


epithelial damage were used (Table 1). These differences in the nomenclature make it however difficult to directly compare the different scoring systems. Less often used parameters in the colitis scoring systems were goblet cell loss, regeneration, muscular and epithelial hyperplasia, edema and the separation between acute and chronic inflammation. In some cases these singularities of the respective scoring system seem to be dependent on the objectives of the study while in most cases the rationale for selection of the parameters was not given.

The number of identified scoring systems for small intestinal disease was significantly lower than for colitis models and had on average lesser citations (Table 1). Two independent scoring systems were identified for intestinal ischemia which include the comprehensible parameters of hyperemia and hemorrhage as well as inflammation and epithelial damage and in the case of the higher cited publication by Park et al. several other more sophisticated parameters $[23,24]$. Only two scoring systems for small intestinal enteritis were detected which both included villous morphology, epithelial damage and inflammation as the main features (Table 1). Surprisingly, only one semiquantitative scoring system for gastritis was identified. Wang et al. scored the severity of Helicobacterinduced gastritis in a uniparametric scoring of five gastric areas. For the sake of completeness this studies was included in this review although it did not fulfill the criterion of multiple parameters [27].

\section{Scoring systems for murine osteoarthritis models}

Seventeen original semiquantitative, multiparametric scoring systems were identified for murine osteoarthritis models (Table 2). Three of these, designated as osteoarthritis in Table 2, are scoring systems for human idiopathic arthritis and were transferred to the murine model to allow for comparisons of the model with the human disease. Of these the the score developed by Mankin et al. has by far the highest citation number which is most

Table 2 Semiquantitative scoring systems for murine osteoarthritis models

\begin{tabular}{|c|c|c|}
\hline Osteoarthritis model & Scoring system: parameters (scale width) & Citations \\
\hline Arthritis [28] & Cartilage structure (0-6), cells (0-3), Safranin-0-stain (0-4), tidemark integrity (0-1) & 1322 \\
\hline Arthritis [30] & Cartilage destruction (0-6), optional subgrading (subdivision in 2 subgrades) & 197 \\
\hline Arthritis [31] & Synovial lining, resident cell density, inflammation (each 0-3) & 49 \\
\hline Collagen-induced [29] & Extent of synovitis, cartilage loss, bone erosions (together $0-3$ ) & 713 \\
\hline Collagen-induced [32] & Inflammation, cartilage destruction, bone erosion (each 0-3) & 273 \\
\hline Collagen-induced [33] & $\begin{array}{c}\text { Infiltration in the exudate, infiltration of the synovial membrane, cartilage destruction, } \\
\text { bone erosion (each } 0-3 \text { ) }\end{array}$ & 198 \\
\hline Collagen-induced [34] & Joint exudate (0-5), proteoglycan depletion (0-3) & 139 \\
\hline Collagen-induced [35] & Bone resorption, inflammation, cartilage damage (each 0-5) & 95 \\
\hline $\begin{array}{l}\text { Mycobacterium butyricum- } \\
\text { induced [36] }\end{array}$ & Synovial thickening, infiltration, pannus formation (each 0-3) & 95 \\
\hline Adjuvant-induced [37] & Percentage of affected area, synovial hyperplasia, cartilage destruction, bone erosion (each 0-3) & 64 \\
\hline IL1-induced arthritis [38] & Synovial infiltration, proteoglycan depletion, cartilage damage (each 0-3) & 41 \\
\hline Instability-induced [39] & Matrix structure, matrix staining, cellularity, subchondral bone (each 0-8) & 85 \\
\hline Instability-induced [40] & Cartilage destruction (0-4), osteophyte formation (0-3) & 85 \\
\hline Hereditary arthritis [41] & Articular cartilage structure (0-8), Toluidine blue-staining (0-6) & 52 \\
\hline Bacterial arthritis [42] & $\begin{array}{l}\text { Infiltration (0-4), pannus formation, cartilage destruction, extra-articular manifestations, } \\
\text { tail lesion (each } 0,1)\end{array}$ & 145 \\
\hline Cartilage repair [43] & $\begin{array}{c}\text { Cell morphology }(0-4) \text {, matrix staining }(0-3) \text {, surface regularity }(0-3) \text {, thickness }(0-2) \text {, } \\
\text { integration of donor cartilage }(0-2)\end{array}$ & 819 \\
\hline Cartilage repair [44] & $\begin{array}{c}\text { Cellular morphology }(0,2,4) \text {, Safranin-O stain }(0-3) \text {, structural regularity }(0-3) \text {, structural integrity }(0-2) \text {, } \\
\text { thickness }(0-2) \text {, bonding }(0-2) \text {, hypocellularity }(0-3) \text {, chondrocyte clustering }(0-2) \text {, } \\
\text { adjacent degeneration (0-3) }\end{array}$ & 297 \\
\hline Cartilage repair [45] & $\begin{array}{l}\text { Relative defect area }(0-4) \text {, integration of repair tissue }(0-3) \text {, matrix staining }(0-4) \text {, } \\
\text { cellular morphology }(0-5) \text {, defect architecture }(0-4) \text {, surface architecture, } \\
\text { percentage of new bone }(0-4) \text {, tidemark formation ( } 0-4)\end{array}$ & 222 \\
\hline Cartilage repair [46] & $\begin{array}{l}\text { Filled depth }(0-4) \text {, integration }(0-2) \text {, surface architecture }(0-3) \text {, cell morphology }(0-3) \text {, } \\
\text { cellularity }(0-2) \text {, tidemark formation }(0-4) \text {, Toloudin blue stain }(0-2)\end{array}$ & 130 \\
\hline Cartilage repair [47] & Defect filling (0-4), osteochondral reconstruction (0-2), matrix staining (0-4), cell morphology (0-4) & 96 \\
\hline
\end{tabular}

IL1, Interleukin 1. 
probably influenced by its common application in the description of human osteoarthritic lesion [28]. The majority of mouse specific systems were established in collageninduced models while models of instability-induced or bacterial arthritis models were only rarely used. Of these, the scoring system by William et al. had the highest citation number [29]. Twelve of the thirteen osteoarthritis scoring systems include an evaluation of cartilage damage, nine included an infiltration parameter, seven included changes in perichondral bone structure and six studies evaluated the extend of synovial infiltration (Table 2). Other parameters like tidemark integrity, proteoglycan content of the cartilage, pannus formation or synovial hyperplasia were used only in the minority or single scoring systems. Again, in most of these descriptions of original scoring systems no rational for inclusion of the respective parameters is given but their usefulness can be comprehended by reflecting the general pathogenesis of osteoarthritis.

\section{Scoring systems for murine renal disease models}

Fourteen original multiparametric, semiquantitative scoring systems for murine models of renal diseases fulfilled the required criteria for inclusion in this review (Table 3). Scoring systems for murine Lupus erythematous models were the dominant model in the category of renal disease models with four appearances. Austin et al. published the lupus nephritis score with the highest citation number [48]. It uses a complex scoring system with 10 parameters and a scale width of four and five respectively and was thus more sophisticated than the other scoring systems which used only four different parameters.

Glomerular cellularity and proliferation were the terms most commonly used in all renal scoring systems except one scoring system for obstructive nephropathy model [61]. In addition, one half of the systems included tubulointerstitial infiltration and fibrosis in the scoring system.

\section{Scoring systems for murine models of neurologic disease}

Twenty-two original scoring systems for murine models of central nervous system (CNS) disease were identified (Table 4). Scoring systems for experimental autoimmune encephalomyelitis (EAE) and stroke clearly dominated results. Due to the wide variety of diseases covered by the system the selection of parameters to be analyzed also had a wide variation and was clearly dependent on the pathophysiology of the disease. But again, the rationale for inclusion of parameters was not consistently given.

A striking feature of CNS disease scoring systems was the relatively low number of ordinal scales for parameter's magnitudes and the common inclusion of multiple anatomical sites into the scoring system (Table 4). This discrepancy is not addressed in the respective publications but may be based on the anatomical diversity of the CNS. Furthermore, the inclusion of absolute values like lesions $/ \mathrm{mm}^{2}$ occurred significantly more often in

Table 3 Semiquantitative scoring systems for murine renal disease models

\begin{tabular}{|c|c|c|}
\hline Renal disease model & Scoring system: parameters (scale width) & Citation \\
\hline Lupus nephritis [48] & $\begin{array}{l}\text { Activity index (glomerular/tubulointerstitial abnormalities (6-tier, each 0-4)); chronicity } \\
\text { index (4-tier, each 0-3) }\end{array}$ & 358 \\
\hline Lupus nephritis [49] & Mesangial thickening, extent of changes (together $0-4$ ) & 44 \\
\hline Lupus nephritis [50] & $\begin{array}{c}\text { Glomerular cell proliferation, lobulation, hyaline droplets, macrophage infiltration } \\
\text { (together } 0-3 \text { ) }\end{array}$ & 25 \\
\hline Lupus nephritis [51] & Glomerular inflammation, proliferation, crescent formation, necrosis (each 0-3) & 1 \\
\hline Toxic nephropathy [52] & Glomerular cellularity, hypertrophy, thrombosis, dilation (together 0-5) & 4 \\
\hline Toxic nephropathy [53] & $\begin{array}{c}\text { Glomerular injury, tubular cysts/casts, podocyte hyperplasia, interstitial } \\
\text { inflammation (each 0-4) }\end{array}$ & 33 \\
\hline Hypertension nephropathy [54] & Mesangial matrix, percentage of glomerular affection (each 0-4) & 524 \\
\hline $\begin{array}{l}\text { Diabetic-/Hypertension-induced } \\
\text { glomerulosclerosis [55] }\end{array}$ & Arteriole hyalinization, glomerular sclerosis (0-4), interstitial volume (\%) & 152 \\
\hline Diabetic nephropathy [56] & Glomerulosclerosis, interstitial fibrosis (each 0-4) & 15 \\
\hline Crescentic glomerulonephritis[57] & $\begin{array}{l}\text { Fibrin deposition, immunoglobulin deposition, tubular damage, } \\
\text { glomerular crescents (each } 0-3 \text { ) }\end{array}$ & 8 \\
\hline $\begin{array}{l}\text { MRL/MPJ mouse } \\
\text { glomerulonephritis [58] }\end{array}$ & Glomerular infiltration, crescents, necrosis, tubular casts, interstitial infiltrates (each 0-4) & 244 \\
\hline Age-associated changes [59] & Mesangial proliferation, sclerosis, hyalinization (together $0-5$ ) & 27 \\
\hline HIV-nephropathy [60] & $\begin{array}{l}\text { Tubuloepithelial degeneration/regeneration, tubular casts, dilation, interstitial infiltration, } \\
\text { glomerular sclerosis, collapse, podocyte hyperplasia (each 0-3) }\end{array}$ & 47 \\
\hline Obstructive nephropathy [61] & Tubular dilation/atrophy, interstitial fibrosis (each 0-3) & 4 \\
\hline
\end{tabular}

HIV, Human immunodeficiency virus; MRL, Murphy Roths Large. 
Table 4 Semiquantitative scoring systems for murine central nervous disease models

\begin{tabular}{|c|c|c|}
\hline Disease model & Scoring system: parameters (scale width) & Citations \\
\hline \multicolumn{3}{|l|}{ Stroke } \\
\hline Focal ischemia [62] & Ischemic neuronal damage (0-3) & 2176 \\
\hline Focal Ischemia[63] & 18 areas $\times$ neuronal injury $(0-5)$ & 23 \\
\hline Global ischemia [64] & Infarcts in 3 cerebral regions (0-4), hippocampus infarction (0-4) & 100 \\
\hline Global ischemia [65] & Eight regions x neuronal cell los/gliosis/iron deposition/gliosis (0-3) & 95 \\
\hline Peripheral nerve ischemia [66] & Edema, fiber regeneration (each 0-4) & 21 \\
\hline \multicolumn{3}{|l|}{ Multiple sclerosis models } \\
\hline EAE [67] & Inflammation, neuronal degeneration (each 0-4) & 160 \\
\hline EAE [68] & Inflammation (cells/cuff), axonal injury, axonal loss (each 0-4) & 81 \\
\hline EAE[69] & Lesion severity, myelin loss/tissue injury, acute inflammation, chronic inflammation (each 0-5) & 79 \\
\hline Theiler's murine encephalomyelitis virus [70] & Neuropil inflammation, demyelination, necrosis, meningeal inflammation (each 0-4) & 77 \\
\hline EAE [71] & Infiltration, demyelination (together 0,1 ) in 16 regions & 64 \\
\hline EAE [72] & Inflammation, necrosis (each 0-3) & 53 \\
\hline EAE [73] & Inflammation (cuffs/100 m²), demyelination (lesions $/ \mathrm{mm}^{2}$ ) & 52 \\
\hline EAE [74] & Meningitis (0-2), perivascular cuffing (0-5), demyelination (0-3) & 43 \\
\hline EAE [75] & Spinal cord demyelination (0-3), inflammatory cells ( $\left.\mathrm{No} . / \mathrm{mm}^{2}\right)$ & 22 \\
\hline \multicolumn{3}{|l|}{ Spinal cord trauma (SCT) } \\
\hline $\mathrm{SCT}[76]$ & Gray matter degeneration/infarction (0-4) in $5 \mu \mathrm{m}$ serial sections & 33 \\
\hline $\mathrm{SCT}[77]$ & $150 \mu \mathrm{m}$ intervals $\times$ Area affected by neuronal degeneration, malacia (0-4) & 23 \\
\hline \multicolumn{3}{|l|}{ Encephalitis/Meningitis } \\
\hline Streptococcus meningitis [78] & Meningeal inflammation $(0-3) \times$ four regions & 46 \\
\hline Trypanosoma encephalitis [79] & Meningitis, perivascular cuffing, neuropil infiltration (each 0-4) & 41 \\
\hline \multicolumn{3}{|l|}{ Miscellaneous CNS diseases } \\
\hline Oxidative damage [80] & 7 areas $x$ necrosis $(0-3)$ & 27 \\
\hline Senescence [81] & Spongiosis (0-3), lipofuscin positive cells (\%) & 23 \\
\hline Spinocerebellar ataxia [82] & Molecular layer thickness, Purkinje cell loss (each 0-3) & 10 \\
\hline
\end{tabular}

$E A E$, Experimental autoimmune encephalomyelitis, SCT, Spinal cord trauma, CNS, Central nervous system.

CNS disease scoring than for other organs although this is not comprehensible in each case. Only one scoring system occurred for a peripheral nerve system disease which has been developed to evaluate peripheral nerve ischemia in a relatively simple two-tier system with a zero to fourscale [66].

\section{Scoring systems for murine models of pulmonary diseases} Fourteen original semiquantitative, multiparametric scoring systems were identified for pulmonary diseases (Table 5). Of these pulmonary fibrosis and pulmonary inflammation were diseases with the highest number scoring systems and citations. For instance, the scoring system developed by Ashcroft, which is a relatively simple multiparametric but single scaled system, is commonly used for the evaluation of lung fibrosis [83]. Three scoring systems were developed for models of general acute lung inflammation (Table 5). They used the parameters of edema and anatomical site specific inflammation as parameters to evaluate the relative amount of inflammatory response. Similar parameters in a wide variety of combinations were used to develop scoring systems for diverse infectious pneumonia models. This variation is again in most cases not based on reasonable argument for the inclusion of a certain parameter in a certain model and therefore not in all cases clearly associated with the supposed pathogen-associated pathogenesis of the respective pneumonia.

\section{Scoring systems for myocardial, vascular and muscular disease models}

Three original scoring systems for the evaluation of viral myocarditis were identified [96-98]. All of them included the evaluation of the parameters of myocardial necrosis and inflammation (Table 6). In addition, two of them also included calcification as a parameter while fibrosis and Evans blue-staining as a marker of myofiber damage were used as a parameter of myocardial disease only once. 
Table 5 Semiquantitative scoring systems for murine models of pulmonary diseases

\begin{tabular}{|c|c|c|}
\hline Pulmonary disease models & Scoring system: parameters (scale width) & Citations \\
\hline Lung fibrosis [83] & Alveolar/bronchial wall thickening, structure distortion, fibrosis (together 0-8) & 273 \\
\hline Lung fibrosis [84] & Fibroblastic foci, established fibrosis, intraalveolar macrophages (each 0-6) & 116 \\
\hline Cystic fibrosis [85] & $\begin{array}{l}\text { Lymphoid infiltrate }(0-5) \text {, goblet cell hyperplasia (0-2), mucus retention }(0-3) \text {, } \\
\text { bronchiolitis }(0-5) \text {, pneumonia }(0-3) \text {, edema (0-2) }\end{array}$ & 101 \\
\hline Ventilator-induced lung injury [86] & $\begin{array}{c}\text { Alveolar congestion, hemorrhage, neutrophils in airspaces/vessel walls, alveolar wall } \\
\text { thickness, hyaline membranes (each 0-4) }\end{array}$ & 78 \\
\hline Pulmonary ischemia [87] & Edema, inflammatory cell infiltration, vascular congestion, alveolar hemorrhage (each 0-3) & 5 \\
\hline Smoke-induced pneumopathy [88] & $\begin{array}{c}\text { Alveolar emphysema, atelectasis, infiltration, hemorrhage, alveolar wall thickness, } \\
\text { perivascular/peribronchiolar edema (each 0-3) }\end{array}$ & 4 \\
\hline Lung inflammation [89] & $\begin{array}{c}\text { Perivascular edema (0-3), perivascular/-bronchiolar inflammation (0-3), } \\
\text { goblet cell metaplasia (0-2) }\end{array}$ & 32 \\
\hline Lung inflammation [90] & $\begin{array}{l}\text { Alvolear wall inflammation, perivenous regions, periarterial/peribronchial regions, } \\
\text { venous/arterial endothelial lesion (each 0-3) }\end{array}$ & 30 \\
\hline Acute lung inflammation [91] & Alveolar necrosis, vascular congestion, infiltration by neutrophils/ macrophages (each 0-4) & 6 \\
\hline RSV pneumonia [92] & $\begin{array}{l}\text { Peribronchiolitis, alveolitis, perivasculitis, hypertrophy of mucus-producing glands, } \\
\text { eosinophilia (each 0-5). }\end{array}$ & 26 \\
\hline Mycoplasma pneumonia [93] & $\begin{array}{l}\text { Quantity/quality of (peri-)bronchial infiltrates, bronchial luminal exudate, perivascular infiltrate, } \\
\text { parenchymal pneumonia (0-3) }\end{array}$ & 56 \\
\hline Pneumocystis carinii infection [94] & Cyst number (0-4), inflammation (0-5) & 87 \\
\hline Streptococcus pneumonia [95] & $\begin{array}{c}\text { Bronchitis, edema, interstitial inflammation, intraalveolar inflammation, pleuritis, } \\
\text { endothelialitis (each 0-4) }\end{array}$ & 0 \\
\hline
\end{tabular}

Three semiquantitative scoring systems for the most important human vascular diseases were identified: atherosclerosis, aneurysms and vasculitis (Table 6) [101-103]. They all cover several aspects of the pathogenesis and pathophysiology of the diseases but have been generally rarely cited yet. The aneurysm scoring system grades the severity of the disease by the extent of medial and adventitial lesion together with the general size of the lesion [102].
The atherosclerosis scoring systems uses a 5-tier system with a 0-1 scale width [102], whereas the vasculitis score uses the parameters infiltration, elastic lamina destruction and intimal thickening, thus indicating that the system may only be useful for evaluation of larger vessel types [103].

Two scoring systems for muscular disease models were identified (Table 6). One recently published scoring system evaluated the extent of Trypanosoma-induced myositis

Table 6 Semiquantitative scoring systems for cardiovascular and muscle disease models

\begin{tabular}{|c|c|c|}
\hline Disease model & Scoring system: parameters (scale width) & Citations \\
\hline \multicolumn{3}{|l|}{ Myocardial diseases } \\
\hline EMCV-induced myocarditis [96] & Myocardial necrosis, infiltration, calcification, fibrosis (together 0-4) & 50 \\
\hline Coxsackievirus-induced myocarditis [97] & Myocardial necrosis, infiltration, calcification (together 0-4) & 33 \\
\hline Coxsackievirus-induced myocarditis [98] & Necrosis, inflammation, Evans blue-stain (each 0-4) & 17 \\
\hline Dilated cardiomyopathy [99] & Myocardial necrosis, fibrosis (together $0-4$ ) & 20 \\
\hline Chronic cardiotoxicity [100] & Qualitative/quantitative myocardial degeneration score (each 0-4) & 72 \\
\hline \multicolumn{3}{|l|}{ Vascular diseases } \\
\hline Aneurysm [101] & Extent of medial, adventitial disruption/size of lesion (together 0-6) & 21 \\
\hline Atherosclerosis [102] & $\begin{array}{l}\text { Medial erosion, foam cells, buried fibrous caps, chondrocyte-like cells, lateral xanthomas } \\
\qquad(\text { each } 0,1)\end{array}$ & 2 \\
\hline Vasculitis [103] & Infiltration, elastic lamina destruction, intimal thickening (together 0-3) & 8 \\
\hline \multicolumn{3}{|l|}{ Muscle diseases } \\
\hline Ischemic necrosis [104] & Infiltration, necrosis, hemorrhage (together 0-10) & 14 \\
\hline Trypanosoma myositis [105] & Number of parasites, eosinophilic infiltration (each 0-3) & 0 \\
\hline
\end{tabular}

EMCV, Encephalomyocarditis virus. 
[104] while the other scoring system was developed to quantify the extent of ischemia-induced muscle necrosis by the parameters necrosis, infiltration and hemorrhage [105].

\section{Scoring systems for hepatic and pancreatic diseases}

Ten original scoring systems for chronic hepatitis have been developed or used for the quantification chronic hepatic disease (Table 7). The scoring systems by Ishak and Knodell are both highly cited scoring systems and cover almost all possible histomorphologic changes in chronically inflamed livers [106,107]. The two identified scoring systems for acute hepatitis quantify lesions by grading the extent of inflammation and necrosis, similar to the Ishak system for chronic hepatitis $[108,109]$.

Five scoring systems for the evaluation of acute pancreatitis have been identified (Table 7). The first and most commonly cited scoring system was published by Schmidt et al. [116]. It uses the five parameters edema, necrosis, inflammation, hemorrhage and fat necrosis to score the extent of pancreatic lesions. All four later developed scoring systems only marginally modified the parameters by omitting a single parameter or including vacuolization as an additional marker (Table 7). In addition, one multiparametric but several uniparametric (data not shown) scoring systems were identified for the quantification of insulitis in mice models. The scoring system by Papaccio et al. uses islet infiltration, atrophy and destruction as parameters for the evaluation of Isle of Langerhans inflammation [122].

\section{Scoring systems for skin and ocular diseases and miscellaneous disease models}

Murine psoriasis models are the only skin disease model with more than one identified scoring system (Table 8 ). Both scoring systems offer a wide variety of parameters for the evaluation of epidermal and dermal changes in models of this relevant human disease [123,124]. In addition, scoring systems for dermal sclerosis, burn scars, atopic dermatitis and epithelial irritation were identified (Table 8).

Five original scoring systems for the evaluation of ocular diseases were identified. Two of these systems use only one parameter for the evaluation of autoimmune and endotoxin uveitis. For the sake of completeness these scoring systems are also displayed in Table 8 , although they do not fulfill requirements for inclusion [133,134]. Furthermore, the identified scoring system for diabetic retinopathy uses two parameters evaluated in absolute numbers of leukocytes per area [130].

Three scoring systems for the evaluation of abdominal adhesions after traumatic or toxic irritation of the peritoneum could be identified. Interestingly, not all scoring

Table 7 Semiquantitative scoring systems for murine hepatic and pancreatic disease models

\begin{tabular}{|c|c|c|}
\hline Disease model & Scoring system: parameters (scale width) & Citations \\
\hline \multicolumn{3}{|l|}{ Hepatic disease } \\
\hline Chronic active hepatitis [107] & Periportal bridging (0-10), intralobular necrosis, portal inflammation, fibrosis (each 0-4) & 2,609 \\
\hline Chronic hepatitis [106] & $\begin{array}{l}\text { Periportal/septal inflammation (0-4), confluent necrosis }(0-6) \text {, focal necrosis/apoptosis/ } \\
\text { inflammation }(0-6) \text {, portal inflammation }(0-4) \text {, fibrosis }(0-6)\end{array}$ & 2,001 \\
\hline Chronic hepatitis [110] & $\begin{array}{l}\text { Mitotic activity, portal inflammation, ductular proliferation, councilman bodies, } \\
\text { fibrosis (each 0-3) }\end{array}$ & 107 \\
\hline Hepatic fibrosis [108] & $\begin{array}{c}\text { Centrolobular vein/perisinusoidal space fibrosis (each } 0-2) \text {, portal tract fibrosis }(0-3) \text {, } \\
\text { septa number }(0-3) \text {, septa width }(0-5)\end{array}$ & 135 \\
\hline Acute hepatitis [111] & Steatosis (0-4), necrosis, inflammation (each 0-2), & 218 \\
\hline Acute hepatitis [109] & Portal/lobular inflammation (each 0-3) & 41 \\
\hline Nutritional hepatopathy [112] & Hepatocyte degeneration, portal inflammation, portal fibrosis (each 0-3) & 25 \\
\hline Alcohol-induced hepatopathy [113] & Steatosis (\%), inflammation/necrosis/fibrosis (each 0-2) & 8 \\
\hline Non-alcoholic Steatohepatitis [114] & Steatosis (0-3), hepatocellular ballooning (0-2), lobular inflammation (0-2) & 1,149 \\
\hline Hepatic ischemia [115] & Location, necrosis (together $0-4$ ) & 12 \\
\hline \multicolumn{3}{|l|}{ Pancreatic disease } \\
\hline Acute pancreatits [116] & Edema, necrosis, inflammation, hemorrhage, fat necrosis (each 0-4) & 307 \\
\hline Acute pancreatitis [117] & Edema, necrosis, inflammation, vacuolization (each 0-4) & 144 \\
\hline Acute pancreatitis [118] & Edema, necrosis, inflammation, hemorrhage (each 0-4) & 89 \\
\hline Acute pancreatitis [119] & Acinar-cell ghosts (\%), acinar cells vacuolization/swelling (\%) & 42 \\
\hline Ischemia-induced acute pancreatitis [120] & Edema, necrosis, infiltration, hemorrhage, vacuolization (each 0-3) & 14 \\
\hline Autoimmune pancreatitis [121] & Infiltration, necrosis, lipomatosis (0-4) & 42 \\
\hline Insulitis [122] & Islet infiltration, destruction, atrophy (0-5) & 49 \\
\hline
\end{tabular}


Table 8 Semiquantitative scoring systems for murine models of eye, skin and miscellaneous diseases

\begin{tabular}{|c|c|c|}
\hline Disease model & Scoring system: parameters (scale width) & Citations \\
\hline \multicolumn{3}{|l|}{ Skin diseases } \\
\hline Burn scars [125] & $\begin{array}{c}\text { Epidermal hyperplasia/hyperkeratosis, hair follicles, apocrine glands, smooth muscles, fibroplasia, } \\
\text { vascular proliferation (each } 0,1 \text { ), collagen orientation }(0-3)\end{array}$ & 26 \\
\hline Systemic sclerosis [126] & Dermal inflammation, thickened collagen bundles, dermal thickness (each 0-3) & 18 \\
\hline Atopic dermatitis [127] & Epidermal hypertrophy, hyperkeratosis, parakeratosis, erosion, inflammation, edema, ulcer (each 0-4) & 8 \\
\hline $\begin{array}{l}\text { UV radiation-induced skin } \\
\text { damage [128] }\end{array}$ & Epidermal thickness (0-3), dermal cellularity (0-3), dermal cyst changes (0-5) & 7 \\
\hline Epithelial irritation [129] & Leukocyte infiltration $(0-5)$, epithelial reaction $(0,1)$ & 1 \\
\hline Psoriasis [123] & $\begin{array}{c}\text { Munro abscesses }(1.5) \text {, hyperkeratosis }(0.5) \text {, parakeratosis }(1) \text {, length of rete ridges }(0.5-1.5) \text {, } \\
\text { lack of granular layer }(1) \text {, acanthosis }(1) \text {, dermis lymphocytic infiltrate }(0.5-1.5) \text {, papillary } \\
\text { papillae congestion (1), thinning above papillae (0.5) }\end{array}$ & 18 \\
\hline Psoriasis [124] & $\begin{array}{l}\text { Epidermal thickness, Stratum corneum thinning, extent of Stratum granulosum/parakeratosis/ } \\
\text { inflammation, microabscesses (each } 0-3 \text { ) }\end{array}$ & 5 \\
\hline \multicolumn{3}{|l|}{ Ocular diseases } \\
\hline Diabetic retinopathy [130] & Inflammation (leukocytes/100 um retina length), leukostasis (leukocytes per vessel lumen) & 25 \\
\hline Oxygen induced retinopathy [131] & $\begin{array}{c}\text { Blood vessel growth, tufts, tortuosity, extraretinal neovascularization, vasoconstriction (each 0-3), } \\
\text { hemorrhage }(0,1)\end{array}$ & 48 \\
\hline Bacterial endophthalmitis [132] & Inflammation, retinal architecture (each $0-4$ ), & 18 \\
\hline Autoimmune uveoretinitis [133] & Infiltration (0-4) & 285 \\
\hline Endotoxin uveitis [134] & Inflammation (0-3) & 47 \\
\hline \multicolumn{3}{|l|}{ Miscellaneous diseases } \\
\hline Abdominal adhesion [135] & Inflammation, fibrosis, necrosis/abscess, granulomas (each 0-3) & 100 \\
\hline Abdominal adhesion [136] & Vessel number, neutrophil infiltration, neutrophils at site (each 0-3) & 4 \\
\hline Abdominal adhesion [137] & Fibrotic matrix, collagen fibers, fibroblast proliferation (together 1-5) & n.r. \\
\hline Embryonic development [138] & Grading $(0-5)$ of 17 parameters & 429 \\
\hline Wound healing [139] & Infiltration, granulation tissue, fibroblasts, collagen deposition (together 0-12) & 369 \\
\hline Thyroiditis [140] & Number of inflammatory foci, parenchymal destruction (together 0-4) & 77 \\
\hline Vaginitis [141] & Epithelial disruption, leucocyte infiltration, edema, vascular injection (each 0-4) & 74 \\
\hline Esophagitis [142] & Epithelial damage/hemorrhage (0-4), inflammation (0-3) & 6 \\
\hline Lymph node [143] & Heterophils, apoptotic histiocytes, sinus histiocytosis, follicular hyperplasia (together 0-5) & 1 \\
\hline Spermatogenic activity [144] & Presence of spermatozoa/spermatides/germ cells/sertoli cells (together 0-10) & n.r. \\
\hline
\end{tabular}

n.r., not reported; UV, ultraviolet light.

systems for abdominal adhesion use fibrosis as a parameter for the grading of the adhesions [135-137]. Finally, very helpful scoring systems for the evaluation of embryonic development and wound healing could be identified (Table 8).

\section{Scoring systems for systemic diseases and transplant rejection}

Three original scoring systems analyzing lesions associated with graft-versus-host disease (GvHD) are available (Table 9). The most cited scoring system by Hill et al. exclusively covers intestinal lesions associated with GvHD [145]. This system allows a very thorough analysis of intestinal lesion using a wide variety of parameters in the small and large intestine. The other two systems also cover intestinal lesions but provide additional parameters for the analysis of hepatic [146] or hepatic and skin lesions [147].

Three original scoring systems have been developed for the analysis lesions associated with hemorrhagic shock (Table 9). The scoring system with the highest citation number only focusses on the pulmonary lesion and offers a variety of parameters for the evaluation of shock-induced lesions in the lung [148]. The other two scoring system also include parameters for lung evaluation but both offer additional parameters for the quantification of intestinal changes $[149,150]$ or in one case offer scoring systems for renal and hepatic lesions [150] associated with hemorrhagic shock.

The identified scoring system for immunotoxicity of toxins more or less demands the analysis of all immune organs but gives a good guideline in terms of the 
Table 9 Semiquantitative scoring systems for systemic disease models

\begin{tabular}{|c|c|c|}
\hline Disease model & Scoring system: parameters (scale width) & Citations \\
\hline \multicolumn{3}{|l|}{ Graft-versus-host disease (GvHD) } \\
\hline Intestinal acute GvHD [145] & $\begin{array}{c}\text { Small intestine: villous blunting, crypt regeneration/apoptosis/loss, enterocyte loss, } \\
\text { infiltration (each 0-4); Colon: crypt regeneration, colonocyte vacuolization, crypt } \\
\text { apoptosis/destruction, infiltration (each 0-4) }\end{array}$ & 389 \\
\hline Acute GvHD [146] & $\begin{array}{l}\text { Small/large bowel: villous blunting, crypt regeneration/apoptosis/loss, luminal sloughing, infiltration, } \\
\text { mucosal ulceration, epithelial/vacuolization (each 0-4), liver: portal infiltration, bile duct apoptosis/ } \\
\text { sloughing, parenchymal apoptosis/abscesses/mitoses, steatosis, cholestasis (each 0-4) }\end{array}$ & 170 \\
\hline Acute GvHD [147] & $\begin{array}{l}\text { Skin: epidermal damage, dermal collagen density, dermal infiltration, subcutaneous fat loss, } \\
\text { hair follicle loss (each 0-2), intestine: crypt apoptosis, inflammation(each 0-4), liver: bile duct } \\
\text { injury (0-4) }\end{array}$ & 50 \\
\hline \multicolumn{3}{|l|}{ Hemorrhagic shock } \\
\hline Lung lesion [148] & $\begin{array}{l}\text { Alveolar membrane thickening, congestion, edema, intraalveolar hemorrhage, interstitial, } \\
\text { intraalveolar infiltration (each 0-3) }\end{array}$ & 36 \\
\hline Intestinal, pulmonary lesions [149] & $\begin{array}{l}\text { Intestine: \% injury (number of edematous villi } \times 0,5+\text { number of villi with epithelial damage } \times 1 \text { ), } \\
\text { lung: number of neutrophils in } 10 \text { fields }\end{array}$ & 2 \\
\hline $\begin{array}{l}\text { Pulmonary, intestinal, renal, } \\
\text { hepatic lesions [150] }\end{array}$ & $\begin{array}{l}\text { Lung: atelectasis, hemorrhage, edema, congestion, inflammation, hyp-eraeration (each 0-3); ileal } \\
\text { mucosal damage, (0-5), liver: congestion, necrosis, vacuolization (each 0-3), Kidney: epithelial } \\
\text { swelling, tubular dilation, necrosis, edema, microthrombosis (each 0-3) }\end{array}$ & 2 \\
\hline \multicolumn{3}{|l|}{ Immunotoxicity } \\
\hline [151] & Complete assessment of all lymphoid organs (each 0-4) & 50 \\
\hline \multicolumn{3}{|l|}{ Transplant rejection } \\
\hline $\begin{array}{l}\text { Banff classification for renal } \\
\text { rejection [152] }\end{array}$ & $\begin{array}{l}\text { Tubulitis, arteritis, mononuclear cell interstitial infiltrates, glomerulitis, interstitial fibrosis, } \\
\text { tubular atrophy, glomerulopathy, mesangial matrix increase, vascular fibrous intimal } \\
\text { thickening, arteriolar hyaline thickening (each 0-3) }\end{array}$ & 1.727 \\
\hline Heart rejection 1990 [153] & Infiltration, myocyte damage (0-4) & 1401 \\
\hline Heart rejection 2005 [154] & Infiltration, myocyte damage (0-3) & 365 \\
\hline Lung rejection [155] & $\begin{array}{l}\text { Inflammation (0-4), lymphocyte infiltration }(0-1) \text {, bronchiolitis obliterans (0-4), Vascular } \\
\text { rejection }(0-1) \text {, vasculitis }(0-1)\end{array}$ & 362 \\
\hline Skin rejection [156] & Acanthosis, ulceration, necrosis, inflammation, granulation tissue (0-5) & 1 \\
\hline
\end{tabular}

GvHD, Graft versus host disease.

nomenclature of morphologic changes associated with the toxin application (Table 9) [151].

Finally, the three scoring systems which have been used for murine transplant rejection models have consistently been developed for the evaluation of tissues from human patients (Table 9). They are all used in mouse models unmodified to allow for better conclusions from the mouse models for the situation in the human patient.

\section{Discussion}

Extensive research of the literature identified 146 originally designed semiquantitative, multiparametric scoring systems for the histopathology of mouse models. These scoring systems cover almost all organs systems and a wide variety of disease models. Colitis and especially ulcerative colitis was the disease model with the largest number of different scoring system closely followed by experimental autoimmune encephalitis (EAE), lupus nephritis and collagen induced osteoarthritis.

The number of citations for the publication including the scoring system varied between few citations and up to 2176 . The citation number clearly reflects the value of the scientific work shown in the papers and thus also indirectly reflects the quality of the included scoring systems. In some cases there is even clear evidence that the high citation number is directly based on the "gold" standard character of the scoring system and its regular use in mouse models or human tissues, for instance the score from Mankin et al. for osteoarthritis evaluation and the scores developed by Knodell and Ishak et al. for chronic hepatitis or the score by Cooper et al. for DSS-colitis $[4,28,106,107]$. Nevertheless, after careful analysis of the publication it also became obvious that scoring systems in publications with a small number of citation also proofed to be of expedience for certain scientific question.

Assuming that the main function of scoring systems is the analysis of the influences of experimental factors on the microscopical tissue morphology the selected parameters should be consciously chosen to be able to reflect the potential changes. The lack of a rationale for the selection of the parameters was therefore an emerging and 
surprising finding during the literature search for this study. Although the selection of parameters in most scoring systems is comprehensibly based on the common knowledge on the pathogenesis of the disease modeled in the mouse, there is only rarely a clear statement or a line of argument for choosing a parameter. Even less often the correlation between scoring parameter value and the magnitude of the clinical symptoms or the differences in the extent of the experimental factor is given as for instance in the excellent study of Cooper et al. [4]. This lack is most probably due to the timeconsuming work involved, but it may however tremendously increase the value and the scientific merit of the scoring system.

\section{Conclusion}

In summary, a final judgment of the quality and the usefulness of the scoring systems presented was not an aim of this study and is after all most probably not possible since the value of a scoring system clearly depends on the scientific question, the underlying hypothesis, the model characteristics and the pathogenesis of the disease. This review may however give an overview on currently available scoring systems and may therefore allow for a better choice for the respective project.

\section{Methods}

\section{Selection of scoring systems}

The systematic review was prepared according to the PRISMA guidelines [157]. All items were considered and can be viewed in Additional file 1. Scoring systems were identified by a comprehensive Pubmed search (http:// www.ncbi.nlm.nih.gov/pubmed/) using a combination of the search terms "mouse", "score", "histopathology". This led to the identification of 1479 publication by October 30, 2012 (Figure 1). Full text versions of all publications were obtained and analyzed for the description of multiparametric, semiquantitative, scoring systems for the histopathology of mouse models. Inclusion of a mouse scoring system in this overview was based on the fulfillment of six parameters.

First, the scoring system had to be based on the semiquantitative evaluation of histopathologic changes in murine tissues. Thus, approaches using digital image analysis for absolute quantification of lesion area, cell number or immunohistochemical signals or scoring systems with dominance of immunohistochemical markers as evaluation parameters were not included.

Second, only scoring systems evaluating more than one histomorphologic parameter were included in the review. Nevertheless, scoring systems with high citation numbers which combined several parameters in a uniparametric score were also included. For instance, if a highly cited scoring system integrated the presence and extent of crypt abscesses, epithelial sloughing and submucosal infiltration into a single score of 0 to 4 the study was also included.

Third, the scoring approach had to be comprehensibly described to allow for reproduction by the reader.

Fourth, the scoring system had to be originally designed for the presented study without citation of former publications. If former publications were cited as the source of the scoring system, the string of citations was followed back to the study originally describing the scoring systems. If scorings systems were not referenced to older studies but similar approaches were detected in earlier publication, only the older study was included in this review.

Fifth, the scoring systems were generally grouped by the organ affected and analyzed. Systemic diseases and transplantation models were included in separate groups. If the number of identified scoring systems for a specific disease model exceeded ten, only the then most cited scoring systems were included in this review.

Citation number was obtained using Thomson Reuters Web Science $^{\odot}$ (http://apps.webofknowledge.com).

\section{Additional file}

Additional file 1: PRISMA 2009 Checklist.

\section{Competing interests}

The author declares that he has no competing interests.

\section{Author's contributions}

RK had the idea of the project, performed the internet search, the data analysis and wrote the abstract.

\section{Acknowledgments}

This study was supported by the FCT grant BD/43731/2008 and the DFG grant KL 2240/1-1. Literature search was tremenduously supported by Camillo Krawczyk, Freie Universität Berlin.

Received: 8 January 2013 Accepted: 19 June 2013

Published: 21 June 2013

\section{References}

1. Renshaw AA, Gould EW: Measuring errors in surgical pathology in real-life practice - Defining what does and does not matter. Am J Clin Pathol 2007, 127(1):144-152.

2. Riber-Hansen $R$, Vainer $B$, Steiniche $T$ : Digital image analysis: a review of reproducibility, stability and basic requirements for optimal results. APMIS 2012, 120(4):276-289.

3. Boyce RW, Dorph-Petersen KA, Lyck L, Gundersen HJG: Design-based Stereology: Introduction to Basic Concepts and Practical Approaches for Estimation of Cell Number. Toxicol Pathol 2010, 38(7):1011-1025.

4. Cooper HS, Murthy SNS, Shah RS, Sedergran DJ: Clinicopathological Study of Dextran Sulfate Sodium Experimental Murine Colitis. Lab Invest 1993, 69(2):238-249.

5. Dieleman LA, Ridwan BU, Tennyson GS, Beagley KW, Bucy RP, Elson CO: Dextran Sulfate Sodium-Induced Colitis Occurs in Severe Combined Immunodeficient Mice. Gastroenterology 1994, 107(6):1643-1652.

6. Dieleman LA, Palmen MJHJ, Akol H, Bloemena E, Pena AS, Meuwissen SGM, van Rees EP: Chronic experimental colitis induced by dextran sulphate sodium (DSS) is characterized by Th1 and Th2 cytokines. Clin Exp Immunol 1998, 114(3):385-391. 
7. Murthy SNS, Cooper HS, Shim H, Shah RS, Ibrahim SA, Sedergran DJ: Treatment of Dextran Sulfate Sodium-Induced Murine Colitis by Intracolonic Cyclosporine. Digest Dis Sci 1993, 38(9):1722-1734.

8. Mahler M, Bristol IJ, Leiter EH, Workman AE, Birkenmeier EH, Elson CO, Sundberg JP: Differential susceptibility of inbred mouse strains to dextran sulfate sodium-induced colitis. Am J Physiol-Gastr L 1998, 274(3):G544-G551.

9. Hudert CA, Weylandt KH, Lu Y, Wang JD, Hong S, Dignass A, Serhan CN, Kang JX: Transgenic mice rich in endogenous omega-3 fatty acids are protected from colitis. P Natl Acad Sci USA 2006, 103(30):11276-11281.

10. Obermeier F, Kojouharoff G, Hans W, Scholmerich J, Gross V, Falk W: Interferon-gamma (IFN-gamma)- and tumour necrosis factor (TNF)induced nitric oxide as toxic effector molecule in chronic dextran sulphate sodium (DSS)-induced colitis in mice. Clin Exp Immunol 1999, 116(2):238-245.

11. Hartmann G, Bidlingmaier C, Siegmund B, Albrich S, Schulze J, Tschoep K, Eigler A, Lehr HA, Endres S: Specific type IV phosphodiesterase inhibitor rolipram mitigates experimental colitis in mice. J Pharmacol Exp Ther 2000, 292(1):22-30.

12. Neurath MF, Fuss I, Kelsall BL, Stuber E, Strober W: Antibodies to Interleukin-12 Abrogate Established Experimental Colitis in Mice. J Exp Med 1995, 182(5):1281-1290.

13. Dohi T, Fujihashi K, Rennert PD, Iwatani K, Kiyono H, McGhee JR: Hapteninduced colitis is associated with colonic patch hypertrophy and $T$ helper cell 2-type responses. J Exp Med 1999, 189(8):1169-1179.

14. Appleyard CB, Wallace JL: Reactivation of Hapten-Induced Colitis and Its Prevention by Antiinflammatory Drugs. Am J Physiol-Gastr L 1995, 269(1):G119-G125

15. Macpherson BR, Pfeiffer CJ: Experimental Production of Diffuse Colitis in Rats. Digestion 1978, 17(2):135-150.

16. ten Hove T, van den Blink B, Pronk I, Drillenburg P, Peppelenbosch MP, van Deventer SJH: Dichotomal role of inhibition of p38 MAPK with SB 203580 in experimental colitis. Gut 2002, 50(4):507-512.

17. Rath HC, Herfarth HH, Ikeda JS, Grenther WB, Hamm TE, Balish E, Taurog JD, Hammer RE, Wilson KH, Sartor RB: Normal luminal bacteria, especially bacteroides species, mediate chronic colitis, gastritis, and arthritis in HLA-B27/human beta(2) microglobulin transgenic rats. J Clin Invest 1996, 98(4):945-953

18. Madsen $\mathrm{KL}$, Doyle JS, Jewell LD, Tavernini MM, Fedorak RN: Lactobacillus species prevents colitis in interleukin 10 gene-deficient mice. Gastroenterology 1999, 116(5):1107-1114.

19. Berg DJ, Davidson N, Kuhn R, Muller W, Menon S, Holland G, ThompsonSnipes L, Leach MW, Rennick D: Enterocolitis and colon cancer in interleukin-10-deficient mice are associated with aberrant cytokine production and CD4(+) TH1-like responses. J Clin Invest 1996, 98(4):1010-1020.

20. Burns RC, Rivera-Nieves J, Moskaluk CA, Matsumoto S, Cominelli F, Ley K: Antibody blockade of ICAM-1 and VCAM-1 ameliorates inflammation in the SAMP-1/Yit adoptive transfer model of Crohn's disease in mice. Gastroenterology 2001, 121(6):1428-1436.

21. Houpt ER, Glembocki DJ, Obrig TG, Moskaluk CA, Lockhart LA, Wright RL Seaner RM, Keepers TF, Wilkins TD, Petri WA: The mouse model of amebic colitis reveals mouse strain susceptibility to infection and exacerbation of disease by CD4(+) T cells. J Immunol 2002, 169(8):4496-4503.

22. Pothoulakis C, Castagliuolo I, Lamont JT, Jaffer A, Okeane JC, Snider RM, Leeman SE: Cp-96,345, a Substance-P Antagonist, Inhibits Rat Intestinal Responses to Clostridium-Difficile Toxin-a but Not Cholera-Toxin. P Nat/ Acad Sci USA 1994, 91(3):947-951.

23. Park PO, Haglund U, Bulkley GB, Falt K: The Sequence of Development of Intestinal Tissue-Injury after Strangulation Ischemia and Reperfusion. Surgery 1990, 107(5):574-580.

24. Lane JS, Todd KE, Lewis MPN, Gloor B, Ashley SW, Reber HA, McFadden DW, Chandler CF: Interleukin-10 reduces the systemic inflammatory response in a murine model of intestinal ischemia/reperfusion. Surgery 1997, 122(2):288-294.

25. de Koning BAE, van Dieren JM, Lindenbergh-Kortleve DJ, van der Sluis M, Matsumoto T, Yamaguchi K, Einerhand AW, Samsom JN, Pieters R, Nieuwenhuis EES: Contributions of mucosal immune cells to methotrexate-induced mucositis. Int Immunol 2006, 18(6):941-949.

26. Stewart-Tull DES, Coote JG, Thompson DH, Candlish D, Wardlaw AC, Candlish A: Virulence spectra of typed strains of Campylobacter jejuni from different sources: a blinded in vivo study. J Med Microbio/ 2009, 58(5):546-553

27. Wang X, Sturegard E, Rupar R, Nilsson HO, Aleljung PA, Carlen B, Willen R, Wadstrom T: Infection of BALB/c a mice by spiral and coccoid forms of Helicobacter pylori. J Med Microbiol 1997, 46(8):657-663.

28. Mankin HJ, Dorfman H, Lippiell L, Zarins A: Biochemical and Metabolic Abnormalities in Articular Cartilage from Osteo-Arthritic Human Hips .2. Correlation of Morphology with Biochemical and Metabolic Data. J Bone Joint Surg Am 1971, 53(3):523.

29. Williams RO, Feldmann M, Maini RN: Antitumor Necrosis Factor Ameliorates Joint Disease in Murine Collagen-Induced Arthritis. P Natl Acad Sci USA 1992, 89(20):9784-9788.

30. Pritzker KPH, Gay S, Jimenez SA, Ostergaard K, Pelletier JP, Revell PA, Salter D, van den Berg WB: Osteoarthritis cartilage histopathology: grading and staging. Osteoarthr Cartilage 2006, 14(1):13-29.

31. Krenn V, Morawietz L, Burmester GR, Kinne RW, Mueller-Ladner U, Muller B, Haupl T: Synovitis score: discrimination between chronic low-grade and high-grade synovitis. Histopathology 2006, 49(4):358-364.

32. Joosten LAB, Helsen MMA, Saxne T, van de Loo FAJ, Heinegard D, van den Berg WB: IL-1 alpha beta blockade prevents cartilage and bone destruction in murine type II collagen-induced arthritis, whereas TNF-alpha blockade only ameliorates joint inflammation. J Immunol 1999, 163(9):5049-5055.

33. Delgado M, Abad C, Martinez C, Laceta J, Gomariz RP: Vasoactive intestinal peptide prevents experimental arthritis by downregulating both autoimmune and inflammatory components of the disease. Nat Med 2001, 7(5):563-568

34. Adkison AM, Raptis SZ, Kelley DG, Pham CTN: Dipeptidyl peptidase I activates neutrophil-derived serine proteases and regulates the development of acute experimental arthritis. J Clin Invest 2002, 109(3):363-371

35. Bendele A, McComb J, Gould T, McAbee T, Sennello G, Chlipala E, Guy M: Animal models of arthritis: Relevance to human disease. Toxicol Pathol 1999, 27(1):134-142.

36. Taniguchi K, Kohsaka H, Inoue N, Terada Y, Ito H, Hirokawa K, Miyasaka N: Induction of the p16(INK4a) senescence gene as a new therapeutic strategy for the treatment of rheumatoid arthritis. Nat Med 1999, 5(7):760-767.

37. Helyes Z, Szabo A, Nemeth J, Jakab B, Pinter E, Banvolgyi A, Kereskai L, Keri G, Szolcsanyi J: Antiinflammatory and analgesic effects of somatostatin released from capsaicin-sensitive sensory nerve terminals in a Freund's adjuvant-induced chronic arthritis model in the rat. Arthritis Rheum 2004, 50(5):1677-1685.

38. Alten $R$, Gram $H$, Joosten LA, van den Berg WB, Sieper J, Wassenberg $S$, Burmester G, van Riel P, Diaz-Lorente M, Bruin GJ, et al: The human anti-IL -1 beta monoclonal antibody ACZ885 is effective in joint inflammation models in mice and in a proof-of-concept study in patients with rheumatoid arthritis. Arthritis Res Ther 2008, 10(3):R67.

39. Rudolphi K, Gerwin N, Verzijl N, van der Kraan P, van den Berg W: Pralnacasan, an inhibitor of interleukin-1 beta converting enzyme, reduces joint damage in two murine models of osteoarthritis. Osteoarthr Cartilage 2003, 11(10):738-746.

40. Kamekura S, Hoshi K, Shimoaka T, Chung U, Chikuda H, Yamada T, Uchida M, Ogata N, Seichi A, Nakamura K, et al: Osteoarthritis development in novel experimental mouse models induced by knee joint instability. Osteoarthr Cartilage 2005, 13(7):632-641.

41. Huebner JL, Hanest MA, Beekman B, TeKoppele JM, Kraus VB: A comparative analysis of bone and cartilage metabolism in two strains of guinea-pig with varying degrees of naturally occurring osteoarthritis. Osteoarthr Cartilage 2002, 10(10):758-767.

42. Bremell T, Abdelnour A, Tarkowski A: Histopathological and Serological Progression of Experimental Staphylococcus-Aureus Arthritis. Infect Immun 1992, 60(7):2976-2985.

43. Wakitani S, Goto T, Pineda SJ, Young RG, Mansour JM, Caplan Al, Goldberg VM Mesenchymal cell-based repair of large, full-thickness defects of articular cartilage. J Bone Joint Surg Am 1994, 76(4):579-592.

44. O'Driscoll SW, Keeley FW, Salter RB: The chondrogenic potential of free autogenous periosteal grafts for biological resurfacing of major fullthickness defects in joint surfaces under the influence of continuous passive motion. An experimental investigation in the rabbit. J Bone Joint Surg Am 1986, 68(7):1017-1035. 
45. Sellers RS, Peluso D, Morris EA: The effect of recombinant human bone morphogenetic protein-2 (rhBMP-2) on the healing of full-thickness defects of articular cartilage. J Bone Joint Surg Am 1997, 79(10):1452-1463.

46. Fortier LA, Mohammed HO, Lust G, Nixon AJ: Insulin-like growth factorenhances cell-based repair of articular cartilage. J Bone Joint Surg Br 2002, 84(2):276-288.

47. Pineda S, Pollack A, Stevenson S, Goldberg V, Caplan A: A semiquantitative scale for histologic grading of articular cartilage repair. Acta Anat (Basel) 1992, 143(4):335-340.

48. Austin HA, Muenz LR, Joyce KM, Antonovych TA, Kullick ME, Klippel JH, Decker JL, Balow JE: Prognostic Factors in Lupus Nephritis - Contribution of Renal Histologic Data. Am J Med 1983, 75(3):382-391.

49. Wang BY, Yamamoto Y, El-Badri NS, Good RA: Effective treatment of autoimmune disease and progressive renal disease by mixed bonemarrow transplantation that establishes a stable mixed chimerism in BXSB recipient mice. P Natl Acad Sci USA 1999, 96(6):3012-3016.

50. Miyazaki T, Ono M, Qu WM, Zhang MC, Mori S, Nakatsuru S, Nakamura Y, Sawasaki T, Endo Y, Nose M: Implication of allelic polymorphism of osteopontin in the development of lupus nephritis in MRL/Ipr mice. Eur J Immunol 2005, 35(5):1510-1520.

51. Keil DE, Peden-Adams MM, Wallace S, Ruiz P, Gilkeson GS: Assessment of trichloroethylene (TCE) exposure in murine strains genetically-prone and non-prone to develop autoimmune disease. J Environ Sci Heal A 2009, 44(5):443-453.

52. Chen SM, Mukoyama T, Sato N, Yamagata SI, Arai Y, Satoh N, Ueda S: Induction of nephrotoxic serum nephritis in inbred mice and suppressive effect of colchicine on the development of this nephritis. Pharmacol Res 2002, 45(4):319-324.

53. Zheng ZY, Schmidt-Ott KM, Chua S, Foster KA, Frankel RZ, Pavlidis P, Barasch J, D'Agati VD, Gharavi AG: A Mendelian locus on chromosome 16 determines susceptibility to doxorubicin nephropathy in the mouse. P Natl Acad Sci USA 2005, 102(7):2502-2507.

54. Raij L, Azar S, Keane W: Mesangial Immune Injury, Hypertension, and Progressive Glomerular Damage in Dahl Rats. Kidney Int 1984, 26(2):137-143.

55. Bader R, Bader H, Grund KE, Mackensenhaen S, Christ H, Bohle A: Structure and Function of the Kidney in Diabetic Glomerulosclerosis Correlations between Morphological and Functional Parameters. Pathol Res Pract 1980, 167(2-4):204-216

56. Yabuki A, Tahara T, Taniguchi K, Matsumoto M, Suzuki S: Neuronal nitric oxide synthase and cyclooxygenase- 2 in diabetic nephropathy of type 2 diabetic OLETF rats. Exp Anim Tokyo 2006, 55(1):17-25.

57. Lai PC, Smith J, Bhangal G, Chaudhry KA, Chaudhry AN, Keith JC, Tam FWK Pusey CD, Cook HT: Interleukin-11 reduces renal injury and glomerular NF-Kappa B activity in murine experimental glomerulonephritis. Nephron Exp Nephrol 2005, 101(4):E146-E154.

58. Watson ML, Rao JK, Gilkeson GS, Ruiz P, Eicher EM, Pisetsky DS, Matsuzawa A, Rochelle JM, Seldin MF: Genetic-Analysis of Mrl-Lpr Mice - Relationship of the Fas Apoptosis Gene to Disease Manifestations and Renal DiseaseModifying Loci. J Exp Med 1992, 176(6):1645-1656.

59. Yumura W, Sugino N, Nagasawa R, Kubo S, Hirokawa K, Maruyama N: Age-Associated Changes in Renal Glomeruli of Mice. Exp Gerontol 1989, 24(3):237-249.

60. Gharavi AG, Ahmad T, Wong RD, Hooshyar R, Vaughn J, Oller S, Frankel RZ, Bruggeman LA, D'Agati VD, Klotman PE, et al: Mapping a locus for susceptibility to HIV-1-associated nephropathy to mouse chromosome 3. P Natl Acad Sci USA 2004, 101(8):2488-2493.

61. Park HC, Yasuda K, Ratliff B, Stoessel A, Sharkovska Y, Yamamoto I, Jasmin JF, Bachmann S, Lisanti MP, Chander $P$, et al: Postobstructive regeneration of kidney is derailed when surge in renal stem cells during course of unilateral ureteral obstruction is halted. Am J Physiol-Renal 2010, 298(2):F357-F364.

62. Pulsinelli WA, Levy DE, Duffy TE: Regional Cerebral Blood-Flow and Glucose-Metabolism Following Transient Forebrain Ischemia. Ann Neurol 1982, 11(5):499-509.

63. Barber PA, Hoyte L, Colbourne F, Buchan AM: Temperature-regulated model of focal ischemia in the mouse: a study with histopathological and behavioral outcomes. Stroke 2004, 35(7):1720-1725.

64. Thoresen M, Bagenholm R, Loberg EM, Apricena F, Kjellmer I: Posthypoxic cooling of neonatal rats provides protection against brain injury. Arch Dis Child 1996, 74(1):F3-F9.

65. Sheldon RA, Sedik C, Ferriero DM: Strain-related brain injury in neonatal mice subjected to hypoxia-ischemia. Brain Res 1998, 810(1-2):114-122.
66. lida H, Schmelzer JD, Schmeichel AM, Wang Y, Low PA: Peripheral nerve ischemia: reperfusion injury and fiber regeneration. Exp Neurol 2003, 184(2):997-1002

67. Fenyk-Melody JE, Garrison AE, Brunnert SR, Weidner JR, Shen F, Shelton BA, Mudgett JS: Experimental autoimmune encephalomyelitis is exacerbated in mice lacking the NOS2 gene. J Immunol 1998, 160(6):2940-2946.

68. Kassis I, Grigoriadis N, Gowda-Kurkalli B, Mizrachi-Kol R, Ben-Hur T, Slavin S, Abramsky O, Karussis D: Neuroprotection and immunomodulation with mesenchymal stem cells in chronic experimental autoimmune encephalomyelitis. Arch Neurol-Chicago 2008, 65(6):753-761.

69. Butterfield RJ, Blankenhorn EP, Roper RJ, Zachary JF, Doerge RW, Teuscher C Identification of genetic loci controlling the characteristics and severity of brain and spinal cord lesions in experimental allergic encephalomyelitis. Am J Pathol 2000, 157(2):637-645.

70. Murray PD, McGavern DB, Lin XQ, Njenga MK, Leibowitz J, Pease LR, Rodriguez M: Perforin-dependent neurologic injury in a viral model of multiple sclerosis. J Neurosci 1998, 18(18):7306-7314.

71. Bright JJ, Du CG, Coon M, Sriram S, Klaus SJ: Prevention of experimental allergic encephalomyelitis via inhibition IL-12 signaling and IL-12 -mediated Th1 differentiation: An effect of the novel anti-inflammatory drug lisofylline. J Immunol 1998, 161(12):7015-7022.

72. Soilu-Hanninen M, Roytta M, Salmi A, Salonen R: Therapy with antibody against leukocyte integrin VLA-4 (CD49d) is effective and safe in virusfacilitated experimental allergic encephalomyelitis. J Neuroimmunol 1997, 72(1):95-105.

73. Zhang J, Li Y, Cui YS, Chen JL, Lu M, Elias SB, Chopp M: Erythropoietin treatment improves neurological functional recovery in EAE mice. Brain Res 2005, 1034(1-2):34-39.

74. Tsunoda I, Kuang LQ, Theil DJ, Fujinami RS: Antibody association with a novel model for primary progressive multiple sclerosis: Induction of relapsing-remitting and progressive forms of EAE in $\mathrm{H} 2(\mathrm{~s})$ mouse strains. Brain Pathol 2000, 10(3):402-418.

75. Lu ZQ, Hu XQ, Zhu CS, Wang DJ, Zheng XP, Liu QT: Overexpression of CNTF in Mesenchymal Stem Cells reduces demyelination and induces clinical recovery in experimental autoimmune encephalomyelitis mice. J Neuroimmunol 2009, 206(1-2):58-69.

76. Sirin BH, Ortac R, Cerrahoglu M, Saribulbul O, Baltalarli A, Celebisoy N, Iskesen I, Rendeci O: Ischaemic preconditioning reduces spinal cord injury in transient ischaemia. Acta Cardiol 2002, 57(4):279-285.

77. Sheng HX, Spasojevic I, Warner DS, Batinic-Haberle I: Mouse spinal cord compression injury is ameliorated by intrathecal cationic manganese (III) porphyrin catalytic antioxidant therapy. Neurosci Lett 2004, 366(2):220-225.

78. Gerber J, Raivich G, Wellmer A, Noeske C, Kunst T, Werner A, Bruck W, Nau R: A mouse model of Streptococcus pneumoniae meningitis mimicking several features of human disease. Acta Neuropathol 2001, 101(5):499-508.

79. Kennedy PGE, Rodgers J, Jennings FW, Murray M, Leeman SE, Burke JM: A substance $P$ antagonist, RP-67,580, ameliorates a mouse meningoencephalitic response to Trypanosoma brucei brucei. P Natl Acad Sci USA 1997, 94(8):4167-4170.

80. Sheldon RA, Jiang XN, Francisco C, Christen S, Vexler ZS, Tauber MG, Ferriero DM: Manipulation of antioxidant pathways in neonatal murine brain. Pediatr Res 2004, 56(4):656-662.

81. Chan YC, Hosoda K, Tsai CJ, Yamamoto S, Wang MF: Favorable effects of tea on reducing the cognitive deficits and brain morphological changes in senescence-accelerated mice. J Nutr Sci Vitaminol (Tokyo) 2006, 52(4):266-273.

82. Oz G, Nelson CD, Koski DM, Henry PG, Marjanska M, Deelchand DK, Shanley R, Eberly LE, Orr HT, Clark HB: Noninvasive detection of presymptomatic and progressive neurodegeneration in a mouse model of spinocerebellar ataxia type 1. J Neurosci 2010, 30(10):3831-3838.

83. Ashcroft T, Simpson JM, Timbrell V: Simple Method of Estimating Severity of Pulmonary Fibrosis on a Numerical Scale. J Clin Pathol 1988, 41(4):467-470.

84. Nicholson AG, Fulford LG, Colby TV, du Bois RM, Hansell DM, Wells AU: The relationship between individual histologic features and disease progression in idiopathic pulmonary fibrosis. Am J Resp Crit Care 2002, 166(2):173-177.

85. Davidson DJ, Dorin JR, Mclachlan G, Ranaldi V, Lamb D, Doherty C, Govan J, Porteous DJ: Lung-Disease in the Cystic-Fibrosis Mouse Exposed to Bacterial Pathogens. Nat Genet 1995, 9(4):351-357. 
86. Nishina K, Mikawa K, Takao Y, Shiga M, Maekawa N, Obara H: Intravenous lidocaine attenuates acute lung injury induced by hydrochloric acid aspiration in rabbits. Anesthesiology 1998, 88(5):1300-1309.

87. Makiuchi A, Yamaura K, Mizuno S, Matsumoto K, Nakamura T, Amano J, Ito Kl: Hepatocyte growth factor prevents pulmonary ischernia-reperfusion injury in mice. J Heart Lung Transp/ 2007, 26(9):935-943.

88. Yang YL, Tang GJ, Wu YL, Yien HW, Lee TS, Kou YR: Exacerbation of wood smoke-induced acute lung injury by mechanical ventilation using moderately high tidal volume in mice. Resp Physiol Neurobi 2008, 160(1):99-108.

89. Zeldin DC, Wohlford-Lenane C, Chulada P, Bradbury JA, Scarborough PE, Roggli V, Langenbach R, Schwartz DA: Airway inflammation and responsiveness in prostaglandin $\mathrm{H}$ synthase-deficient mice exposed to bacterial lipopolysaccharide. Am J Respir Cell Mol Biol 2001, 25(4):457-465.

90. Curtis JL, Byrd PK, Warnock ML, Kaltreider HB: Requirement of Cd4-Positive T-Cells for Cellular Recruitment to the Lungs of Mice in Response to a Particulate Intratracheal Antigen. J Clin Invest 1991, 88(4):1244-1254.

91. Eveillard M, Soltner C, Kempf M, Saint-Andre JP, Lemarie C, Randrianarivelo C, Seifert H, Wolff M, Joly-Guillou ML: The virulence variability of different Acinetobacter baumannii strains in experimental pneumonia. J Infection 2010, 60(2):154-161.

92. Barends M, van Oosten M, de Rond CGH, Dormans JAMA, Osterhaus ADME, Neijens HJ, Kimman TG: Timing of infection and prior immunization with respiratory syncytial virus (RSV) in RSV-enhanced allergic inflammation. J Infect Dis 2004, 189(10):1866-1872.

93. Cimolai N, Taylor GP, Mah D, Morrison BJ: Definition and Application of a Histopathological Scoring Scheme for an Animal-Model of Acute Mycoplasma-Pneumoniae Pulmonary Infection. Microbiol Immunol 1992, 36(5):465-478.

94. Beck JM, Warnock ML, Curtis JL, Sniezek MJ, Arraj-Peffer SM, Kaltreider HB, Shellito JE: Inflammatory responses to Pneumocystis carinii in mice selectively depleted of helper T lymphocytes. Am J Respir Cell Mol Biol 1991, 5(2):186-197.

95. Lammers AJ, de Porto AP, de Boer OJ, Florquin S, van der Poll T: The role of TLR2 in the host response to pneumococcal pneumoniain absence of the spleen. BMC Infect Dis 2012, 12(1):139.

96. Dong R, Liu P, Wee L, Butany J, Sole MJ: Verapamil Ameliorates the Clinical and Pathological Course of Murine Myocarditis. J Clin Invest 1992, 90(5):2022-2030.

97. Hiraoka Y, Kishimoto C, Kurokawa M, Ochiai H, Sasayama S: Effects of Polyethylene-Glycol Conjugated Superoxide-Dismutase on Coxsackievirus B3 Myocarditis in Mice. Cardiovasc Res 1992, 26(10):956-961.

98. Wang YX, Da Cunha V, Vincelette J, White K, Velichko S, Xu Y, Gross C, Fitch RM, Halks-Miller M, Larsen BR, et al: Antiviral and myocyte protective effects of murine interferon-beta and -alpha 2 in coxsackievirus B3-induced myocarditis and epicarditis in Balb/c mice. Am J Physiol-Heart C 2007, 293(1):H69-H76.

99. Kanda T, Araki M, Nakano M, Imai S, Suzuki T, Murata K, Kobayashi I: Chronic Effect of Losartan in a Murine Model of Dilated Cardiomyopathy Comparison with Captopril. J Pharmacol Exp Ther 1995, 273(2):955-958.

100. Rahman A, More N, Schein PS: Doxorubicin-Induced Chronic Cardiotoxicity and Its Protection by Liposomal Administration. Cancer Res 1982, 42(5):1817-1825.

101. Ghazalpour A, Wang XP, Lusis AJ, Mehrabian M: Complex inheritance of the 5-lipoxygenase locus influencing atherosclerosis in mice. Genetics 2006, 173(2):943-951.

102. Bennett BJ, Wang SS, Wang XP, Wu XH, Lusis AJ: Genetic Regulation of Atherosclerotic Plaque Size and Morphology in the Innominate Artery of Hyperlipidemic Mice. Arteriosd Throm Vas 2009, 29(3):348-A318.

103. Yamada A, Miyazaki T, Lu LM, Ono M, Ito MR, Terada M, Mori S, Hata K, Nozaki Y, Nakatsuru S, et al: Genetic basis of tissue specificity of vasculitis in MRL/Ipr mice. Arthritis Rheum 2003, 48(5):1445-1451.

104. Carter WO, Bull C, Bortolon E, Yang L, Jesmok GJ, Gundel RH: A murine skeletal muscle ischemia-reperfusion injury model: differential pathology in BALB/C and DBA/2N mice. J App/ Physiol 1998, 85(5):1676-1683.

105. Nascentes GAN, Meira WSF, Lages-Silva E, Ramirez LE: Immunization of Mice with a Trypanosoma cruzi-Like Strain Isolated from a Bat: Predictive Factors for Involvement of Eosinophiles in Tissue Damage. Vector-Borne Zoonot 2010, 10(10):989-997.

106. Ishak K, Baptista A, Bianchi L, Callea F, De Groote J, Gudat F, Denk H, Desmet V, Korb G, MacSween RN, et al: Histological grading and staging of chronic hepatitis. J Hepatol 1995, 22(6):696-699.
107. Knodell RG, Ishak KG, Black WC, Chen TS, Craig R, Kaplowitz N, Kiernan TW, Wollman J: Formulation and Application of a Numerical Scoring System for Assessing Histological Activity in Asymptomatic Chronic Active Hepatitis. Hepatology 1981, 1(5):431-435.

108. Chevallier M, Guerret S, Chossegros P, Gerard F, Grimaud JA: A histological semiquantitative scoring system for evaluation of hepatic fibrosis in needle liver biopsy specimens: comparison with morphometric studies. Hepatology 1994, 20(2):349-355.

109. Siegmund B, Lear-Kaul KC, Faggioni R, Fantuzzi G: Leptin deficiency, not obesity, protects mice from Con A-induced hepatitis. Eur J Immunol 2002, 32(2):552-560.

110. VanNieuwkerk CMJ, Elferink RPJO, Groen AK, Ottenhoff R, Tytgat GNJ, Dingemans KP, Weerman MAVB, Offerhaus GJA: Effects of ursodeoxycholate and cholate feeding on liver disease in FVB mice with a disrupted mdr2 P-glycoprotein gene. Gastroenterology 1996, 111(1):165-171.

111. Nanji AA, Khettry U, Sadrzadeh SMH: Lactobacillus Feeding Reduces Endotoxemia and Severity of Experimental Alcoholic Liver (Disease). P Soc Exp Biol Med 1994, 205(3):243-247.

112. Curran TJ, Uzoaru I, Das JB, Ansari G, Raffensperger JG: The Effect of Cholecystokinin-Octapeptide on the Hepatobiliary Dysfunction Caused by Total Parenteral-Nutrition. J Pediatr Surg 1995, 30(2):242-247.

113. Sancho-Bru P, Bataller R, Fernandez-Varo G, Moreno M, Ramalho LN, Colmenero J, Mari M, Claria J, Jimenez W, Arroyo V, et al: Bradykinin attenuates hepatocellular damage and fibrosis in rats with chronic liver injury. Gastroenterology 2007, 133(6):2019-2028.

114. Kleiner DE, Brunt EM, Van Natta M, Behling C, Contos MJ, Cummings OW, Ferrell LD, Liu YC, Torbenson MS, Unalp-Arida A, et al: Design and validation of a histological scoring system for nonalcoholic fatty liver disease. Hepatology 2005, 41(6):1313-1321.

115. Cavalieri B, Perrelli MG, Aragno M, Ramadori P, Poli G, Cutrin JC: Ischaemic preconditioning modulates the activity of Kupffer cells during in vivo reperfusion injury of rat liver. Cell Biochem Funct 2003, 21(4):299-305.

116. Schmidt J, Rattner DW, Lewandrowski K, Compton CC, Mandavilli U, Knoefel WT, Warshaw AL: A Better Model of Acute-Pancreatitis for Evaluating Therapy. Ann Surg 1992, 215(1):44-56.

117. Rongione AJ, Kusske AM, Kwan K, Ashley SW, Reber HA, McFadden DW: Interleukin 10 reduces the severity of acute pancreatitis in rats. Gastroenterology 1997, 112(3):960-967.

118. Kusske AM, Rongione AJ, Ashley SW, McFadden DW, Reber HA: Interleukin10 prevents death in lethal necrotizing pancreatitis in mice. Surgery 1996, 120(2):284-289.

119. Cuzzocrea S, Mazzon E, Dugo L, Serraino I, Centorrino T, Ciccolo A, Van de Loo FAJ, Britti D, Caputi AP, Thiemermann C: Inducible nitric oxide synthase-deficient mice exhibit resistance to the acute pancreatitis induced by cerulein. Shock 2002, 17(5):416-422.

120. Dembinski A, Warzecha Z, Ceranowicz P, Dembinski M, Cieszkowski J, Pawlik WW, Tomaszewska R, Konturek SJ, Konturek PC: Effect of ischemic preconditioning on pancreatic regeneration and pancreatic expression of vascular endothelial growth factor and platelet-derived growth factor-A in ischemia/reperfusion-induced pancreatitis. J Physiol Pharmacol 2006, 57(1):39-58.

121. Kanno H, Nose M, Itoh J, Taniguchi Y, Kyogoku M: Spontaneous Development of Pancreatitis in the Mrl/Mp Strain of Mice in Autoimmune Mechanism. Clin Exp Immunol 1992, 89(1):68-73.

122. Papaccio G, Nicoletti F, Pisanti FA, Bendtzen K, Galdieri M: Prevention of spontaneous autoimmune diabetes in NOD mice by transferring in vitro antigen-pulsed syngeneic dendritic cells. Endocrinology 2000, 141(4):1500-1505.

123. Baker BS, Brent L, Valdimarsson H, Powles AV, Alimara L, Walker M, Fry L: Is Epidermal-Cell Proliferation in Psoriatic Skin-Grafts on Nude-Mice Driven by T-Cell Derived Cytokines. Brit J Dermatol 1992, 126(2):105-110.

124. Kvist PH, Svensson L, Hagberg O, Hoffmann V, Kemp K, Ropke MA: Comparison of the effects of vitamin $D$ products in a psoriasis plaque test and a murine psoriasis xenograft model. J Trans/ Med 2009, 7:107.

125. Singer AJ, Thode HC, McClain SA: Development of a histomorphologic scale to quantify cutaneous scars after burns. Acad Emerg Med 2000, 7(10):1083-1088.

126. Yamamoto T, Takagawa S, Katayama I, Mizushima Y, Nishioka K: Effect of superoxide dismutase on bleomycin-induced dermal sclerosis: Implications for the treatment of systemic sclerosis. J Invest Dermatol 1999, 113(5):843-847. 
127. Watanabe T, Hamada K, Tategaki A, Kishida H, Tanaka H, Kitano M, Miyamoto T: Oral Administration of Lactic Acid Bacteria Isolated from Traditional South Asian Fermented Milk 'Dahi' Inhibits the Development of Atopic Dermatitis in NC/Nga Mice. J Nutr Sci Vitaminol (Tokyo) 2009, 55(3):271-278.

128. Lee HJ, Kim JS, Song MS, Seo HS, Moon CJ, Kim JC, Jo SK, Jang JS, Kim SH: Photoprotective Effect of Red Ginseng against Ultraviolet Radiationinduced Chronic Skin Damage in the Hairless Mouse. Phytother Res 2009, 23(3):399-403

129. Hjortsjo C, Saxegaard E, Young A, Dahl JE: In vivo and in vitro irritation testing of low concentrations of hydrofluoric acid. Acta Odontol Scand 2009, 67(6):360-365

130. Gubitosi-Klug RA, Talahalli R, Du YP, Nadler JL, Kern TS: 5-lipoxygenase, but not 12/15-lipoxygenase, contributes to degeneration of retinal capillaries in a mouse model of diabetic retinopathy. Diabetes 2008, 57(5):1387-1393.

131. Higgins RD, Yu K, Sanders RJ, Nandgaonkar BN, Rotschild T, Rifkin DB: Diltiazem reduces retinal neovascularization in a mouse model of oxygen induced retinopathy. Curr Eye Res 1999, 18(1):20-27.

132. Ramadan RT, Ramirez R, Novosad BD, Callegan MC: Acute inflammation and loss of retinal architecture and function during experimental Bacillus endophthalmitis. Curr Eye Res 2006, 31(11):955-965.

133. Caspi RR, Roberge FG, Chan CC, Wiggert B, Chader GJ, Rozenszajn LA, Lando Z, Nussenblatt RB: A New Model of Autoimmune-Disease Experimental Autoimmune Uveoretinitis Induced in Mice with 2 Different Retinal Antigens. J Immunol 1988, 140(5):1490-1495.

134. Cousins SW, Guss RB, Howes EL, Rosenbaum JT: Endotoxin-Induced Uveitis in the Rat - Observations on Altered Vascular-Permeability, Clinical Findings, and Histology. Exp Eye Res 1984, 39(5):665-676.

135. Tyrell J, Silberman H, Chandrasoma P, Niland J, Shull J: Absorbable Versus Permanent Mesh in Abdominal Operations. Surg Gynecol Obstet 1989, 168(3):227-232.

136. Corona R, Verguts J, Schonman R, Binda MM, Mailova K, Koninckx PR: Postoperative inflammation in the abdominal cavity increases adhesion formation in a laparoscopic mouse model. Fertil Steril 2011, 95(4):1224-1228.

137. Suga H, Teraoka S, Ota K, Komemushi S, Furutani S, Yamauchi S, Margolin S: Preventive effect of pirfenidone against experimental sclerosing peritonitis in rats. Exp Toxicol Pathol 1995, 47(4):287-291.

138. Brown NA, Fabro S: Quantitation of Rat Embryonic-Development Invitro a Morphological Scoring System. Teratology 1981, 24(1):65-78.

139. Greenhalgh DG, Sprugel KH, Murray MJ, Ross R: Pdgf and Fgf Stimulate Wound-Healing in the Genetically Diabetic Mouse. Am J Pathol 1990, 136(6):1235-1246

140. Braley-Mullen H, Sharp GC, Medling B, Tang HW: Spontaneous autoimmune thyroiditis in NOD.H-2h4 mice. J Autoimmun 1999, 12(3):157-165.

141. Eckstein P, Jackson MCN, Millman N, Sobrero AJ: Comparison of Vaginal Tolerance Tests of Spermicidal Preparations in Rabbits and Monkeys. J Reprod Fertil 1969, 20(1):85.

142. Poplawski C, Sosnowski D, Szaflarska-Poplawska A, Sarosiek J, McCallum R, Bartuzi Z: Role of bile acids, prostaglandins and COX inhibitors in chronic esophagitis in a mouse model. World J Gastroentero 2006, 12(11):1739-1742.

143. Bondarenko A, Hewicker-Trautwein M, Erdmann N, Angrisani N, Reifenrath J, Meyer-Lindenberg A: Comparison of morphological changes in efferent lymph nodes after implantation of resorbable and non-resorbable implants in rabbits. Biomed Eng Online 2011, 10:32.

144. Johnsen SG: Testicular biopsy score count a method for registration of spermatogenesis in human testes. Normal values and results in 335 hypogonadal males. Hormones 1970, 1:2-25.

145. Hill GR, Crawford JM, Cooke KR, Brinson YS, Pan LY, Ferrara JLM: Total body irradiation and acute graft-versus-host disease: The role of gastrointestinal damage and inflammatory cytokines. Blood 1997, 90(8):3204-3213.

146. Cooke KR, Hill GR, Crawford JM, Bungard D, Brinson YS, Delmonte J, Ferrara $J$ LM: Tumor necrosis factor-alpha production to lipopolysaccharide stimulation by donor cells predicts the severity of experimental acute graft-versus-host disease. J Clin Invest 1998, 102(10):1882-1891.

147. Kaplan DH, Anderson BE, McNiff JM, Jain D, Shlomchik MJ, Shlomchik WD: Target antigens determine graft-versus-host disease phenotype. J Immunol 2004, 173(9):5467-5475.

148. Murao $Y$, Loomis W, Wolf $P$, Hoyt DB, Junger WG: Effect of dose of hypertonic saline on its potential to prevent lung tissue damage in a mouse model of hemorrhagic shock. Shock 2003, 20(1):29-34.
149. Ahmadi-Yazdi C, Williams B, Oakes S, Moore FD: Attenuation of the Effects of Rat Hemorrhagic Shock with a Reperfusion Injury-Inhibiting Agent Specific to Mice. Shock 2009, 32(3):295-301.

150. Mees ST, Gwinner M, Marx K, Faendrich F, Schroeder J, Haier J, Kahlke V: Influence of sex and age on morphological organ damage after hemorrhagic shock. Shock 2008, 29(6):670-674.

151. Kuper CF, Harleman JH, Richter-Reichelm HB, Vos JG: Histopathologic approaches to detect changes indicative of immunotoxicity. Toxicol Pathol 2000, 28(3):454-466.

152. Racusen LC, Solez K, Colvin RB, Bonsib SM, Castro MC, Cavallo T, Croker BP, Demetris AJ, Drachenberg CB, Fogo AB, et al: The Banff 97 working classification of renal allograft pathology. Kidney Int 1999, 55(2):713-723.

153. Billingham ME, Cary NR, Hammond ME, Kemnitz J, Marboe C, McCallister HA, Snovar DC, Winters GL, Zerbe A: A working formulation for the standardization of nomenclature in the diagnosis of heart and lung rejection: Heart Rejection Study Group. The International Society for Heart Transplantation. J Heart Transplant 1990, 9(6):587-593.

154. Stewart S, Winters GL, Fishbein MC, Tazelaar HD, Kobashigawa J, Abrams J, Andersen CB, Angelini A, Berry GJ, Burke MM, et al: Revision of the 1990 working formulation for the standardization of nomenclature in the diagnosis of heart rejection. J Heart Lung Transp/ 2005, 24(11):1710-1720.

155. Yousem SA, Berry GJ, Brunt EM, Chamberlain D, Hruban RH, Sibley RK, Stewart S, Tazelaar HD: A Working Formulation for the Standardization of Nomenclature in the Diagnosis of Heart and Lung Rejection - Lung Rejection Study-Group. J Heart Transplant 1990, 9(6):593-601.

156. Gaucher S, Nicco C, Jarraya M, Batteux F: Viability and Efficacy of Coverage of Cryopreserved Human Skin Allografts in Mice. Int J Low Extr Wound 2010, 9(3):132-140.

157. Moher D, Liberati A, Tetzlaff J, Altman DG: Preferred reporting items for systematic reviews and meta-analyses: the PRISMA statement. J Clin Epidemiol 2009, 62(10):1006-1012

doi:10.1186/1746-6148-9-123

Cite this article as: Klopfleisch: Multiparametric and semiquantitative scoring systems for the evaluation of mouse model histopathology - a systematic review. BMC Veterinary Research 2013 9:123.

\section{Submit your next manuscript to BioMed Central and take full advantage of:}

- Convenient online submission

- Thorough peer review

- No space constraints or color figure charges

- Immediate publication on acceptance

- Inclusion in PubMed, CAS, Scopus and Google Scholar

- Research which is freely available for redistribution

Submit your manuscript at www.biomedcentral.com/submit
C Biomed Central 